\title{
SUPPLEMENTARY MATERIAL FOR REACTIVE POINT PROCESSES: A NEW APPROACH TO PREDICTING POWER FAILURES IN UNDERGROUND ELECTRICAL SYSTEMS
}

\author{
By Şeyda Ertekin, Cynthia Rudin and Tyler H. McCormick \\ Massachusetts Institute of Technology and the University of Washington
}

1. Related work. A general linear self-exciting process is identified by the conditional intensity function

$$
\lambda(t)=\mu+k_{0} \sum_{t_{k}<t} g\left(t-t_{k}\right)
$$

where $\mu$ is the baseline hazard rate. The Hawkes Process (Hawkes, 1971a,b) is a particularly tractable self-exciting point process with an exponential kernel, developed for earthquake modeling. Historically, a lot of effort has gone into modeling earthquakes, e.g., works of Ogata $(1988,1998)$, which consider Epidemic-Type-Aftershock-Sequences (ETAS). The formulation and parametrization of the ETAS model divides the effect of earthquakes into two components, namely background and aftershock events. Background events, corresponding to $\mu$ in the expression above, occur independently according to a stationary Poisson process. An earthquake elevates the risk of aftershocks, where the magnitude and temporal properties of this elevated risk are determined by the kernel $g$. Subsequent works incorporated the spatial distance of past seismic events for modeling the seismic activity at a point of interest (Musmeci and Vere-Jones, 1992; Ogata, 1998) (note that spatial distance elements do not appear to be first order effects for manhole event prediction).

In recent applications of SEPP's, Mohler et al. (2011) described a SEPP model that is based on the space-time ETAS model for modeling the rate of crimes. In particular, they drew a parallel between the spread of seismic activity and crime behavior, and proposed a model for residential burglaries that captures the spatial-temporal clustering patterns observed in crime data. Egesdal et al. (2010) studied a similar problem of modeling gang rivalries, where an event involving rival gangs can lead to retaliatory (self-excitatory) acts of violence. The same problem was addressed by Louie, Masaki and Allenby (2010), which extends the model of Egesdal et al. (2010) to incorporate the spatial influence of past events (similar to the space-time ETAS model of Ogata, 1998). Lewis 
et al. (2010) analyzed temporal patterns of civilian death reports in Iraq between from 2003 until 2007 and defined a SEPP model with an exponential kernel similar to the one defined by Egesdal et al. (2010). The paper mainly focuses on modeling the background violence rate $\mu$ since exogenous factors such as political decisions, changes in troop levels, etc., can have a significant impact on the rate of events.

In the financial world, SEPP models have been used both at micro and macro scales. Engle and Russell (1998) proposed a SEPP model for analyzing transaction data on individual stocks and how they are spread over a given time interval. At the macro scale, contagion of financial crises was modeled as a mutually exciting jump process by Aït-Sahalia, Cacho-Diaz and Laeven (2010). They modeled the dynamics of asset returns and how they fluctuate based on financial events in the same region (self-excitation) as well as in other regions (mutual excitation). Note that usually when handling self-exciting processes, the "multivariate setting" (as described by Embrechts, Liniger and Lin, 2011) has the marks (where excitation occurs) as multivariate. In our case there is only one dimension: vulnerability level. In our model, we have multiple manholes, each with their own multidimensional vector of features. These features influence all of the marks for that manhole.

Recently, self-exciting point processes have been shown to be effective for genome analysis (Reynaud-Bouret and Schbath, 2010), preventative maintenance for water pipes (Yan et al., 2013), as well as analyzing and capturing the dynamics and neural spike trains (Krumin, Reutsky and Shoham, 2010). These domains have distinct properties that require unique models (for example, the self-excitation component of the model for DNA sequences replaces geographic distance with a distance between basepairs).

In the context of social networks, Crane and Sornette (2008) studied endogenous and exogenous factors that influence the dynamics of viewing activity for YouTube videos. They argue that an individual's viewing behavior is influenced by their communication with others about what to watch next. Based on this assumption, their SEPP model defines the baseline rate $\mu$ to capture spontaneous views that are not triggered by epidemic effects on the network. The self-excitation component is controlled by the number of potential viewers influenced directly by person $i$ who viewed a video at time $t_{i}$, and a "memory" kernel that captures the waiting time distribution for the influenced individuals (the time between finding out about a video and viewing the video). A similar model with a memory kernel has been used to model conversation event sequences (Masuda 
et al., 2012).

RPPs differ from all of these related models in several ways: the form of the model is different, where the excitation is a proportion of the baseline rate, the saturation functions are unique to RPP's, and the external inspections are not present in other models.

We know of no previous works using SEPP's for power grid maintenance. We remark that for manhole event prediction, it is not clear whether the self-exciting property that we observe is due to a weakening in the infrastructure caused by previous events (in analogy with aftershocks of earthquakes) or whether it is due to a weakening in the infrastructure caused by another (hidden) source.

2. Inspection Policy for Simulated Data. The inspection policy used in the simulated data consists of three components. These components are similar to those within Con Edison's current inspection policy.

- Targeted inspections for the most vulnerable manholes: We created periodic inspections at regularly scheduled intervals for the most vulnerable $k$ manholes. Specifically, every 50 days, we inspected the top $10 \%$ of vulnerable manholes for that day, all at once. While this targeting policy is effective in terms of reducing the overall hazard rate, it is plausible that some manholes may be inspected over and over whereas others can go un-inspected for extended periods of time, which violates current government safety regulations. Hence we require regular inspections for all manholes, discussed just below.

- Targeted inspections for all manholes: We ensure that all manholes are regularly maintained even if they have not had a serious event (or not selected by the first component) for a long time. At each day, inspections are scheduled for a small subset of manholes that have not been inspected for a long time (200 days). We schedule inspections for (up to) $1 \%$ of manholes for each day using this policy.

- Ad hoc inspections: Con Edison's ad hoc inspections program allows workers that are in a manhole (for instance to install new service or make repairs) to fill in an inspections form while they are on the premises. We model these ad hoc inspections as being uniformly randomly distributed, where at each day, we randomly select $0.2 \%$ of the manholes and inspect them. 
If, from the combination of these different policies, there is more than one inspection scheduled on the same day, only one inspection will be performed that day.

3. Conditional Frequency (CF) Estimator for the RPP. For this method, we locate event/inspection "trails" in the time-series data for each manhole. We define a trail as a sequence of time steps of fixed granularity (days, weeks, etc.) for a specific manhole where a predefined pattern of inspection/event records exists. The trail ends when we observe an inspection or event record. We estimate the model parameters in a case-controlled way, using only the applicable trails, in the following order:

1. Estimate $\lambda_{0}$. The baseline hazard rate $\lambda_{0}$ refers to the likelihood of observing an event under steady-state conditions, for a manhole that has no previous event record in the database. That is, at a given time $t$ where there has not been a recorded inspection or event, $\lambda_{0}$ is the probability of an event at that time. Computation of $\hat{\lambda}_{0}$ requires trails that start from the earliest recorded time until the observation of the very first event record or inspection record for each manhole. If the manhole never had an event or inspection, the trail is the full time course. Let $S_{t}$ denote the number of manholes that have survived without an event up to and including time $t-1$, and did not have an inspection through time $t$. Let $E_{t}$ denote the number of these manholes that had an event at time $t$. We first compute an estimate of the baseline event rate $\lambda_{0}$ for each $t$ separately as $E_{t} / S_{t}$. The later times represent trails that are long, and there are much fewer such trails. Thus we have far less data at later times than at earlier times, and the estimates at later times can be unreliable. For our estimate of $\lambda_{0}$ we take an average of these estimates, weighted by the number of observations for each estimate, $S_{t}$. It reduces to a pooled average as follows:

$$
\hat{\lambda}_{0} \approx \frac{\sum_{t=1}^{T_{\max }}\left(E_{t} / S_{t}\right) \cdot S_{t}}{\sum_{t=1}^{T_{\max }} S_{t}}=\frac{\sum_{t=1}^{T_{\max }} E_{t}}{\sum_{t=1}^{T_{\max }} S_{t}}
$$

where $T_{\max }$ is the length of the longest trail that we are willing to consider.

To validate the $\mathrm{CF}$ estimator method result, we were able to reproduce the value of $\lambda_{0}$ given in a simulation very closely. In simulation studies described in more detail in the supplementary material, we estimated $\hat{\lambda}_{0}=0.0097$, when the true value was $\lambda_{0}=0.01$.

As an alternative method for estimating $\lambda_{0}$ in a personalized way, one can use a long-term probabilistic model (such as the one we had developed for the project previously in [Rudin 
et al. (2010)]) that incorporates features for each manhole.

2. Estimate $C_{1}$. After finding the baseline hazard rate, we estimate $C_{1}$ from trails in the data where the effects of $g_{2}$ and $g_{4}$ are hypothesized to have approximately vanished, and where the manhole had a prior event. In other words, the trails that we consider have the following properties: $i$ ) there was a past event in the manhole, $i i$ ) the start of the trail is a time at which the effect of a previous event/inspection is fully decayed, and $i i i$ ) the end of the trail is the observation of an event or inspection record in that manhole if there was one. There are some manholes for which we have multiple trails by these criteria, and we use all of them. Defining $E_{\Delta t}$ and $\bar{E}_{\Delta t}$ as the total number of trails in the data with and without an event after $\Delta t$ of the start of the trail (and no inspections during the trail), we again use a pooled average to get:

$$
\begin{aligned}
\hat{\lambda}_{0}\left(1+\hat{C}_{1}\right) & =\frac{\sum_{\Delta t=1}^{T} E_{\Delta t}}{\sum_{\Delta t=1}^{T}\left(E_{\Delta t}+\bar{E}_{\Delta t}\right)} \\
\hat{C}_{1} & =\left[\frac{1}{\hat{\lambda}_{0}} \frac{\sum_{\Delta t=1}^{T} E_{\Delta t}}{\sum_{\Delta t=1}^{T}\left(E_{\Delta t}+\bar{E}_{\Delta t}\right)}\right]-1 .
\end{aligned}
$$

3. Estimate $g_{2}$ : To obtain the CF estimates, we consider events that are observed at steadystate, meaning that the effect of previous events/inspections, if any, should have fully decayed. In other words, we choose trails starting at events that occurred at baseline vulnerability levels. Furthermore, to keep the computation consistent, we consider only trails where there was already a previous serious event in that manhole (so that we can consistently use $\hat{C}_{1}$ in our computations). The trail ends at the immediate next event/inspection record, if any. Redefining $E_{\Delta t}$ and $\bar{E}_{\Delta t}$ as the total number of trails in the database with and without an event after $\Delta t$ of the start of the trail (and no inspections within that time), we compute an estimated failure rate $\frac{E_{\Delta t}}{E_{\Delta t}+\bar{E}_{\Delta t}}$ for each $\Delta t$ interval and a CF estimate for $g_{2}$ at time $\Delta t$ :

$$
g_{2}^{\text {CFestimator }}(\Delta t)=\frac{1}{\lambda_{0}} \frac{E_{\Delta t}}{E_{\Delta t}+\bar{E}_{\Delta t}}-\hat{C}_{1}-1 \text {. }
$$

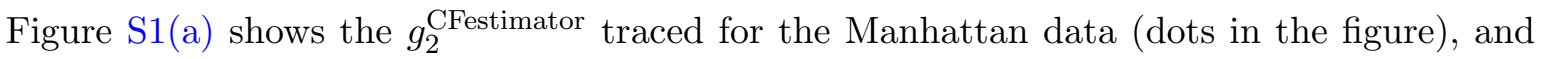
the parameterized $g_{2}$ function we fitted afterwards. The fitted curve uses the form given in (2) in the main paper. We also evaluated the shape of $g_{2}$ curves estimated using this method using data simulated with known $g_{2}$ curves. The results, given in detail in the supplementary material Section 3.1, indicate that this method accurately recovers the true $g_{2}$ function. 


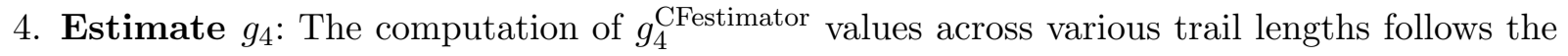
same procedure as for $g_{2}^{\text {CFestimator }}$, with the distinction that the trails now start with an inspection record. That is, we use only trails where the manhole was inspected at the baseline vulnerability level and there was a history of at least one serious event in that manhole. Figure S2(d) in the supplementary material shows that we are able to recover $g_{4}$ for such trails in the simulated data. We cannot well-estimate $g_{4}$ for the Manhattan dataset since the inspection program is relatively new, and since the effect of an inspection and repair can take much longer to wear off than the effect of an event (in fact, longer than the span of the data that we have presently). Engineers at Con Edison assisted us to estimate $g_{4}$ using domain knowledge, with a band of uncertainty that we take into account in Section 6 in the main paper.

5. Estimate $g_{1}$ : The computation of $g_{1}$ involves focusing on the trails with event cascades; that is, we are interested in the trails that start with a serious event that is under the influence of a prior serious event. These are events for which a previous event's effect has not fully decayed. These cascades of multiple events close together in time "activate" the $g_{1}$ component of our model.

Accordingly, we find trails that start at the time of a serious event that closely follows at least one prior serious event. These trails end at the next event (when a new trail begins) or at the next inspection. We exclude trails under the influence of a past inspection. We first discretize possible values for $\left(\sum g_{2}\right)$ into bins. At each timestep of each trail, we will determine which is the corresponding bin, then estimate a probability of event for each bin. We denote a trail as $T r$, its start time as time $(T r)$, and $E_{\Delta t}^{T r}=\left\{e_{1}, e_{2}, \ldots, e_{k}\right\}$ as the set of previous recent events whose effects have not completely decayed at time $(\mathrm{Tr})+\Delta t$. This includes the event at time $(T r)$. At each timestep $\Delta t$ after the start of trail $T r$, we compute

$$
V^{T r}=\sum_{e \in E_{\Delta t}^{T r}} g_{2}(\operatorname{time}(T r)+\Delta t-\operatorname{time}(e))
$$

We then determine which bin $V^{T r}$ corresponds to, which is denoted by $b$. Depending on whether or not we observed a serious event at time $\left(T_{i}\right)+\Delta t$, we add 1 to either $S_{\text {Event }, b}$ or $S_{\text {NoEvent }, b}$. Once $S_{\text {Event }, b}$ and $S_{\text {NoEvent }, b}$ have been computed using all timesteps for all trails, the value of $g_{1}$ in bin $b$ equals $S_{\text {Event }, b} /\left(S_{\text {Event }, b}+S_{\text {NoEvent }, b}\right)$.

For the Manhattan dataset, our estimate of $g_{1}$ was afterwards parameterized using the func- 


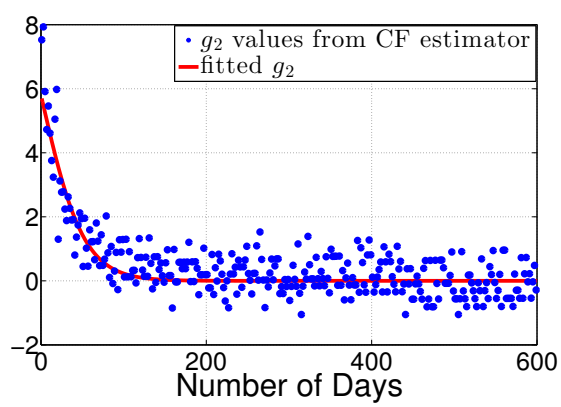

(a) Function $g_{2}^{\text {CFestimator }}$ and fitted $g_{2}$

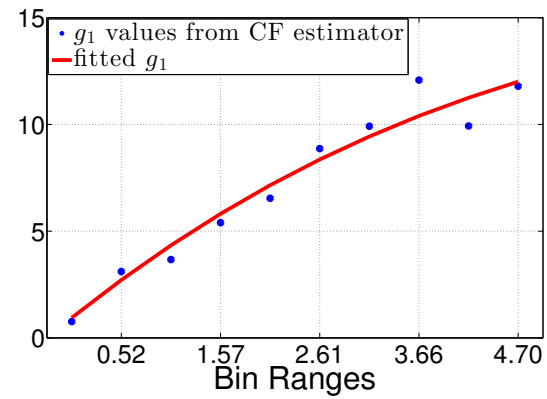

(b) Function $g_{1}^{\text {CFestimator }}$ and fitted $g_{1}$

FiG S1. Fitted $g_{2}$ and $g_{1}$ functions for the Manhattan dataset.

tional form in (2) in the main paper, as shown in Figure S1(b).

6. Estimate $g_{3}$ : The computation of $g_{3}$ follows the same steps defined for $g_{1}$, with the distinction that we now consider trails that start with an inspection where that inspection is still under the influence of an earlier inspection, and no previous events.

The estimates for the parameters this procedure yields for the Manhattan power data are given in Section 2 in the main paper.

3.1. Experiments for the Conditional Frequency (CF) Estimator for the RPP: Verification on Simulated Data. To evaluate the CF estimator for the RPP with simulated data, we simulated manhole events and inspections (according to the policy provided in Supplementary Materials Section 2) for 10,000 manholes over 10,000 days. We used $\lambda_{0}=0.01$ as the baseline vulnerability and set $C_{1}=0.1$. The model functions for the simulation were specified as:

$$
\begin{aligned}
& g_{1}(t)=1.5 \times\left(1-\log \left(1+e^{-t}\right) \times \frac{1}{\log (2)}\right) \\
& g_{3}(t)=0.9 \times\left(1-\log \left(1+e^{2 t}\right) \times \frac{1}{\log (2)}\right) \\
& g_{2}(t)=\frac{1}{1+e^{\beta t}} \\
& g_{4}(t)=\frac{-1}{1+e^{\gamma t}} .
\end{aligned}
$$

with $\beta=0.1$ and $\gamma=0.1$. In the resulting simulated dataset, we have 1.1 million serious events and 700 thousand inspection records.

In this experiment, we are interested in assessing whether the resulting event/inspection records for the simulated manholes can be used to infer the underlying generative process. The functions 


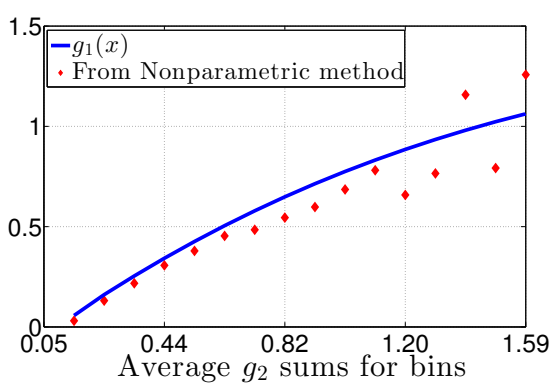

(a) $g_{1}$

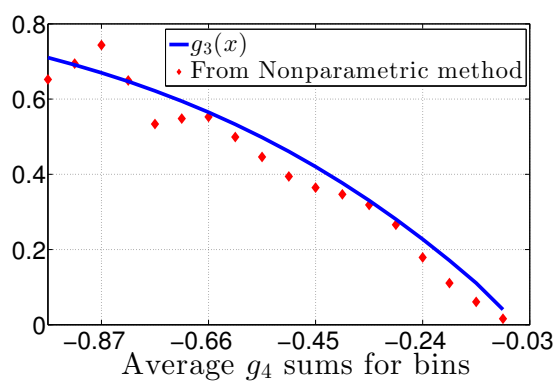

(c) $g_{3}$

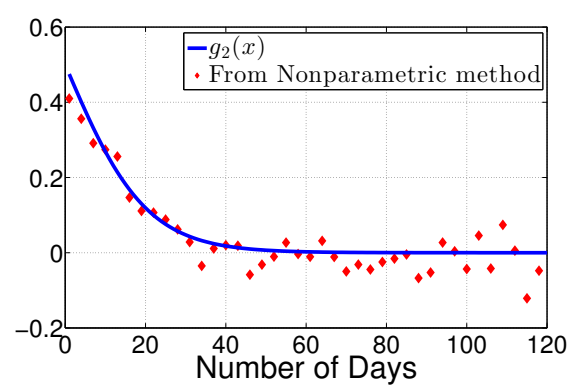

(b) $g_{2}$

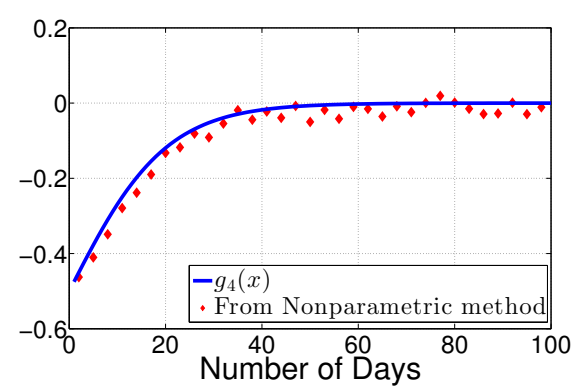

(d) $g_{4}$

FIG S2. Results for the CF estimator approach experiments with simulated data. Blue curves are the data we wish to recover from the simulations, written in equations (1). Red dots are from the CF estimator approach.

$g_{1}, g_{2}, g_{3}$ and $g_{4}$ from the simulated model functions above were plotted with blue curves in Figure $\mathrm{S} 2$, and the results from the $\mathrm{CF}$ estimator for the RPP were plotted with red dots. The red dots from the CF estimator for the RPP, particularly those derived from averages over many points, are close to the underlying simulated values.

The quality of the CF estimator approach fades as the amount of time between manhole events (far right of the x-axis for the $g_{2}$ plot) grows beyond our usual collection window. For $g_{2}$, the CF estimator for the RPP can be used to create points as far out as 120 days, but the number of points in each bin for this many days out can be small. This happens also in reality, where we do not have much data for when the spacing between manhole events becomes extremely large. Similarly for the large values of $g_{1}$, there are not that many days within the simulated data where the sum of $g_{2}$ reaches very large values. This would only happen when several manhole events occur within a very small period of time, which is unlikely. 


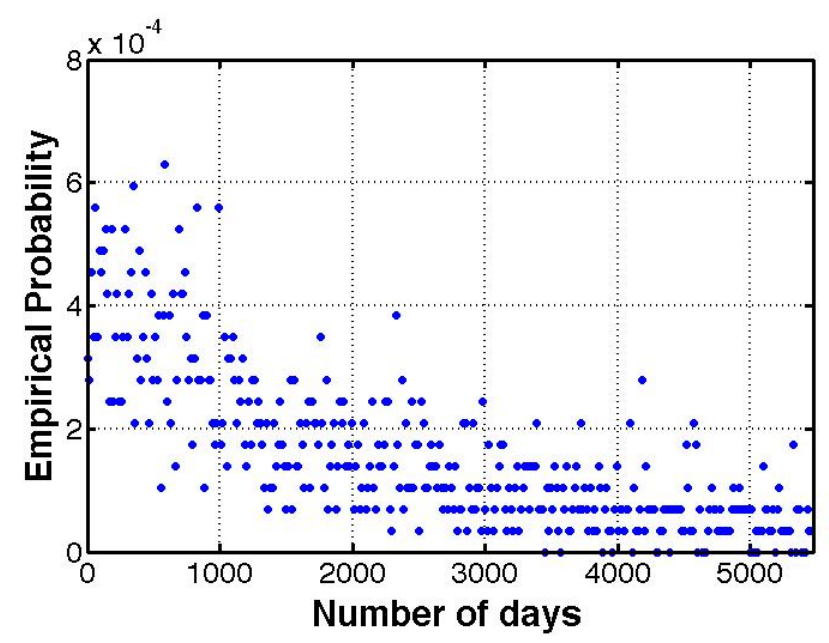

FIG S3. The distribution of inter-arrival times of events according to the random effects model.

4. Analysis of Manhattan Data using Random Effects Model. We investigate whether the empirical event probabilities that we observe in Figure 3(a) in the main paper are more likely to be a consequence of self-exciting behavior or if they can be explained by a simple random effects model instead. As it turns out, the behavior is not easily explained by a natural random effects model.

In order to check the random effects hypothesis, we count the total number of events for each manhole and assume the events at each manhole are distributed according to a Poisson distribution with $\lambda_{i}=\frac{N_{i}}{Z}$, where $N_{i}$ is the number of events at manhole $i$ and $Z$ is the total number of days. Then, for each event in manhole $i$, we sample an inter-arrival time from the exponential distribution with parameter $\lambda_{i}$. Figure S3 shows the empirical probability of inter-arrival times considering all events in all manholes. The rate of decay we observe with this hypothesis is significantly slower than what we observe in Figure 3(a) in the main paper. Because of this, the observations are more likely to be explained using a self-exciting model.

5. Likelihood-based Experiments. In this section we describe a procedure for fitting the RPP model using maximum likelihood. As described in the manuscript, the likelihood is that of a non-homogenous Poisson Process. To find the maximum likelihood estimate, we sampled the coefficients $\boldsymbol{v}$ from a normal distribution. Figure S4 displays the region of maximum likelihood (shown in dark red), where the data have the highest likelihood with respect to the sampled values of $\boldsymbol{v}$. We could have also used optimization techniques to find the maximum, though we found 

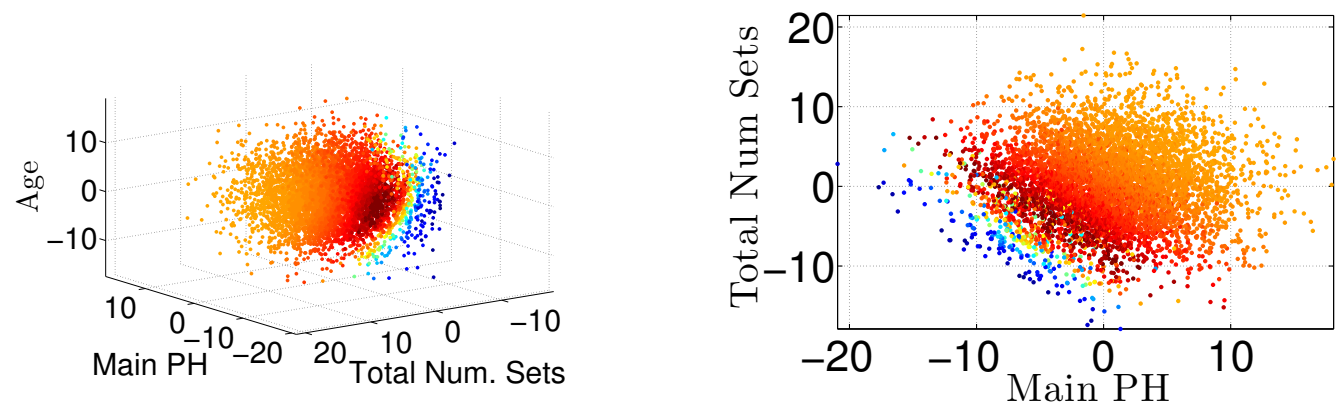

FIG S4. Log Likelihood for Manhattan Dataset. Each axis represents possible values of a coefficient. The "Age" axis is for the age of the oldest cable set within the manhole, "Main PH" axis for the number of main phase cables in the manhole and "Total Num. Sets" axis is for the total number of cable sets including main, service, and streetlight cables.

this approach to be sufficient. The covariates are the number of main phase cables in the manhole (number of current carrying cables between two manholes), the total number of cable sets (total number of bundles of cables) including main, service, and streetlight cables, and the age of the oldest cable set within the manhole. All covariates were normalized to be between -0.5 and 0.5.

5.1. Verification of Maximum Likelihood Method on Simulated Data. Here we attempt to recover the $\beta, \gamma$ combination used for simulating data, using the likelihood-based RPP. We simulated 1,000 manholes over 10,000 days with $\lambda_{0}=0.01, C_{1}=0.1$ (assumed to be known), with $g_{2}$ and $g_{4}$ provided in (1) with $\beta$ and $\gamma$ set at 0.05 for $g_{2}$ and $g_{4}$, respectively, and $g_{1}$ and $g_{3}$ as the identity. We employed the same inspection policy as described in Supplementary Materials Section 2.

Figure S5 plots the log likelihood values computed from the simulated data for various $\beta, \gamma$ combinations. Figure S5(a) is the fitted surface plot. Figure S5(b) shows the same surface plot, viewed from a projection onto the $\beta$ axis. A projection onto the $\beta$ axis in Figure S5(c) shows that the $\beta$ achieving the maximum likelihood is close to the simulated (ideal) value of $\beta=0.05$. Figure S5(d) plots the likelihood as a function of $\gamma$, for the cross-section that includes only $\beta$ values within the range $\beta=[0.045,0.055]$. Again, we observe the peak of the likelihood near 0.05 , in agreement with the latent $\gamma$ parameter that we used to generate the dataset.

6. Bayesian RPP Experiments: Verification on Simulated Data. This set of experiments assesses whether we can reliably estimate the decay parameters $\beta$ and $\gamma$ using Approximate Bayesian Computation (ABC). The underlying data we are trying to recover is simulated, and also 


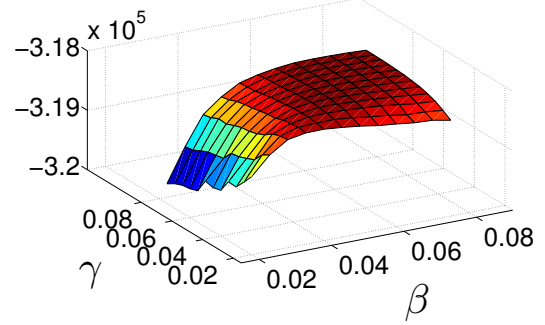

(a) Log Likelihood

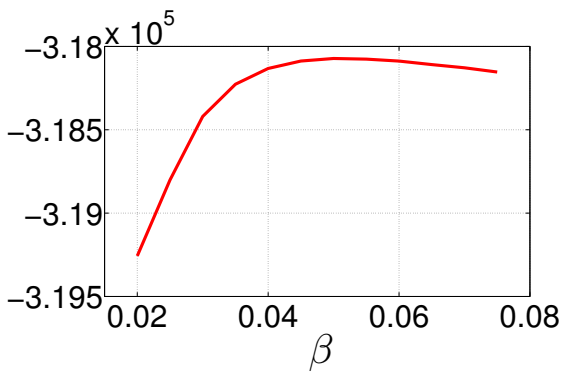

(c) Log Likelihood $\beta$-axis

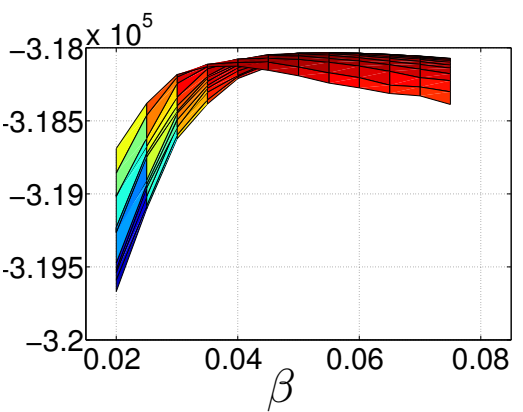

(b) Log Likelihood

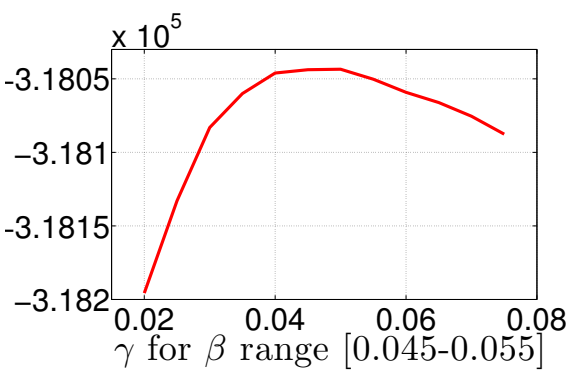

(d) Log Likelihood $\gamma$-cross section

FIG S5. Log Likelihood for Simulated Data

$\mathrm{ABC}$ requires simulated data. To be clear in this section, we will call the data we are trying to recover "recovery data," and the data generated using ABC we will call "ABC-simulated data." What we hope is that the statistics of the $\mathrm{ABC}$ simulated data at the correct parameter values will match the summary statistics of the recovery data, in a way that is observable. We conducted experiments in two stages, as follows:

Events only: In the first stage, the recovery data included simulated events but no inspections, and $g_{1}$ was set to be the identity. In this setting, the search space is one-dimensional (along the $\beta$-axis) and our vulnerability model for the recovery data becomes

$$
\lambda=\lambda_{0}\left(1+\sum_{\forall t<t_{e}} g_{2}\left(t-t_{e}\right)+C_{1} \mathbb{1}_{\left[\exists t_{e}<t\right]}\right) .
$$

We used the $g_{2}$ in (1) with $\beta=0.06$. The parameters were set to $\lambda_{0}=0.02, C_{1}=0.1$, which are assumed to be known. We generated recovery data for 1,000 manholes over 10,000 days.

To test the ABC algorithm, we assumed the form of $g_{2}$ in (1) and attempted to recover $\beta$. We randomly sampled $\beta$ values uniformly from the range $[0.02,0.1]$, and for each value sampled we calculated the KL and DNE summary statistics of the ABC-simulated data, and compared them to those of the recovery data, as outlined in Section 3.3. Recall that KL measures the KL 


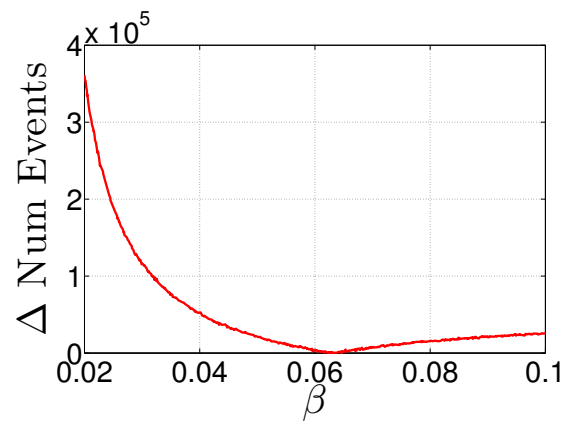

(a) Number of Simulated Events.

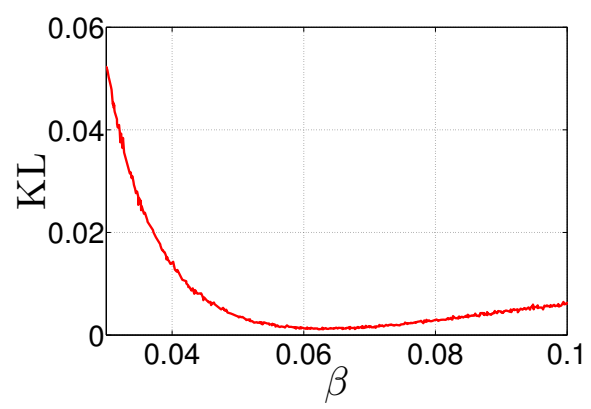

(b) KL Divergence

FIG S6. Results for $A B C$ with Events.

divergence between the distributions of time differences between events, and DNE measures the difference in the total number of events between the recovered event sequences and the ABCsimulated event sequences. Figures S6(a) and S6(b) show that the ABC implementation with either summary statistic is able to recover the latent value of $\beta=0.06$.

Events and Inspections: When we introduce inspections into the simulated data (using the inspection policy in Supplementary Materials Section 2), the search space now spans the $\gamma$-axis as well as the $\beta$ axis. The recovery data uses the following model:

$$
\lambda=\lambda_{0}\left(1+\sum_{\forall t<t_{e}} g_{2}\left(t-t_{e}\right)+\sum_{\forall t<t_{i}} g_{4}\left(t-t_{i}\right)+C_{1} \mathbb{1}_{\left[\exists t_{e}<t\right]}\right),
$$

where $g_{2}$ and $g_{4}$ are of the form in (1) with $\beta=\gamma=0.05$, we have known values $\lambda_{0}=0.01, C_{1}=0.1$, and $g_{1}$ and $g_{3}$ are the identity. We again followed the inspection policy in Supplementary Materials Section 2. The recovery data were simulated for 1,000 manholes and 10,000 days.

We conducted $\mathrm{ABC}$ simulations for uniformly randomly sampled $\beta$ and $\gamma$ values, both from within the range $[0.025,0.075]$. Figures $\mathrm{S} 7(\mathrm{a})$ and $\mathrm{S} 7(\mathrm{~b})$ show the full surface plot of KL and DNE measures as a function of ABC-simulated $\beta$ and $\gamma$ values. Projecting onto the $\beta$ axis, Figures S7(c) and $\mathrm{S} 7(\mathrm{~d})$ show that both KL and DNE are minimized in close proximity of $\beta=0.05$. Similarly, taking a cross section where $\beta$ values are in the range [0.045, 0.055], we see from Figures $\mathrm{S} 7(\mathrm{e})$ and $\mathrm{S} 7(\mathrm{f})$ that the $\gamma$ value of 0.05 from the recovery data is not far from the minimizer for either the KL or DNE metric. 


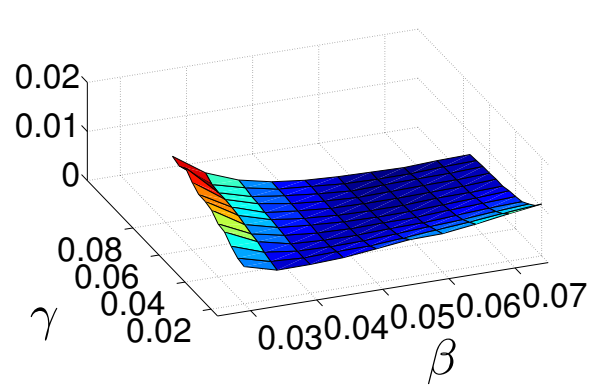

(a) KL Divergence

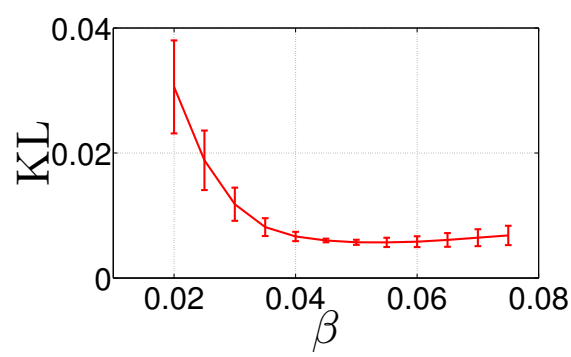

(c) KL Divergence - $\beta$

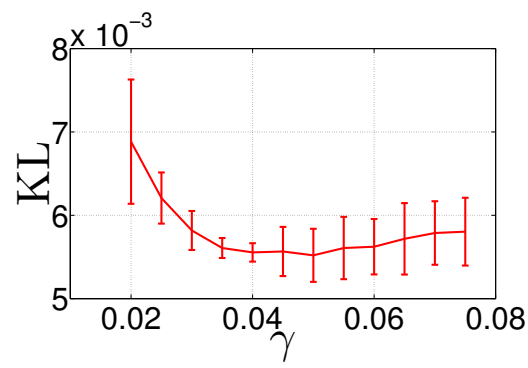

(e) KL Divergence - $\gamma$

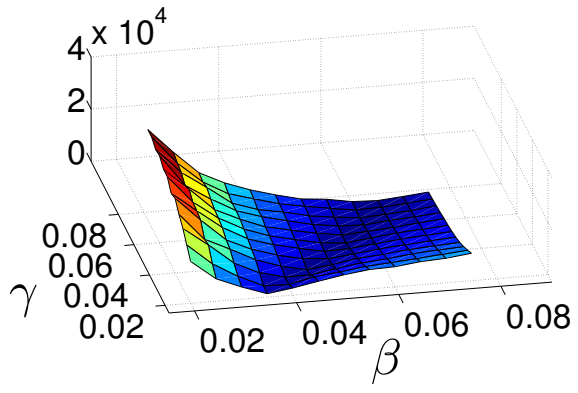

(b) Number of Simulated Events.

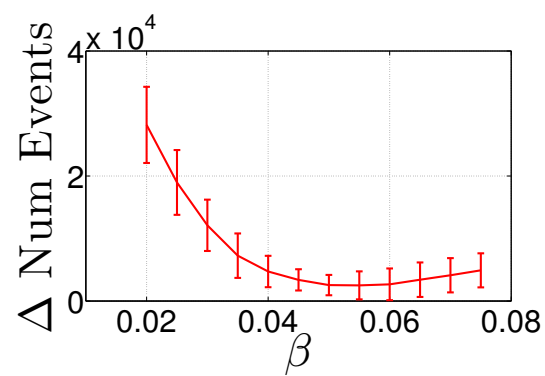

(d) Number of Simulated Events - $\beta$

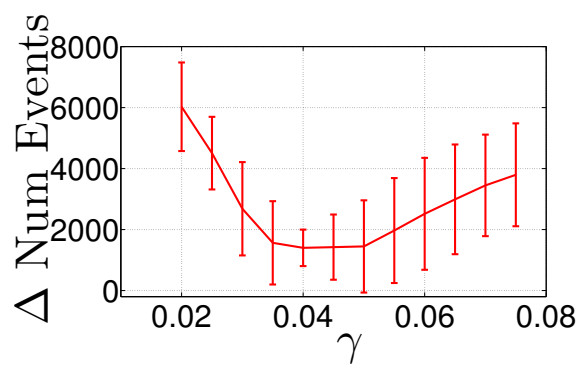

(f) Number of Simulated Events - $\gamma$

FIG S7. Results for ABC with Events and Inspections.

7. Importance sampling algorithm. In our application we were most interested in the posterior mode. If one would like to reconstruct the full posterior instead, this could be accomplished for our model using a straightforward importance sampling algorithm. We present such and algorithm in Figure S8. Using the prior as the proposal density, one would simply need to choose an appropriate kernel, $K(\cdot)$ and bandwidth (typically through cross-validation).

8. Simulated Dataset for the NYC Power Grid. We provide a publicly available dataset that has similar characteristics of the NYC grid dataset that we used in this paper. The dataset is generated based on the simulation of events and inspections in 53,525 manholes over a period of 15 years. It is currently available at https://github.com/ElectricGrid/Dataset 
- Inputs. Data $\left\{t_{1}^{(p)}, \ldots, t_{N_{E}^{(p)}}^{(p)}\right\}_{p}$ and $\left(\left\{\bar{t}_{1}^{(p)}, \ldots, \bar{t}_{N_{I}^{(p)}}^{(p)}\right\}_{p}, \mathbf{M}\right)$; a summary function $S(\cdot)$; a sampling distribution for parameter values $\pi$.

- Initialize. Set $s_{o b s}=S\left(\left\{t_{1}^{(p)}, \ldots, t_{N_{E}^{(p)}}^{(p)}\right\}_{p}\right)$.

- Iterate. For iter $=1, \ldots$, Iter,

1. Choose parameter values randomly from proposal distribution $g$.

2. Simulate a sequence of event times $\left\{t_{1}^{(p), \text { iter }}, \ldots, t_{N_{E}^{\text {iter, }(p)}}^{(p), \text { iter }}\right\}_{p}$ and inspection times using the chosen parameter values. Compute $s_{\text {iter }}=S\left(\left\{t_{1}^{(p), \text { iter }}, \ldots, t_{N_{E}^{(p), \text { iter }}}^{(p), \text { iter }}\right\}_{p}\right)$.

3. Define weights $w_{i}=\pi / g$ where $\pi$ is the prior.

- Outputs. $\left|s_{\text {iter }}-s_{\text {obs }}\right|$ for iter $=1, \ldots$, Iter and weights.

- For each $i$, set $w_{i}=\pi / g$ with probability $K\left(\left(s_{i t e r}-s_{o b s}\right) / h\right)$ for kernel $K$ and bandwidth $h$ and 0 otherwise.

FIG S8. An ABC importance sampling algorithm. The parameters might be $\beta$ and $\gamma$ when there are no covariates, or $\boldsymbol{v}$ and $\boldsymbol{\omega}$ when covariates are present. Note that in our application we simulate proposals from the prior distribution and thus have importance weights of one.

- Manhole Characteristics: Each manhole is characterized by two covariates; the number of main phase $(\mathrm{PH})$ cables, and the age of oldest cable set. Following the histograms of these covariates in the Con Edison dataset as shown in the manuscript Section 2, we generated these two covariates for the simulated manholes by drawing samples from gamma and uniform distributions, respectively. For the number of main phase cables, we chose parameters for the gamma distribution by fitting them to the Manhattan data, as shown in Figure S9. We have shape parameter $k=1.7041$ and scale parameter $\theta=12.0141$. For the age of oldest cable set,

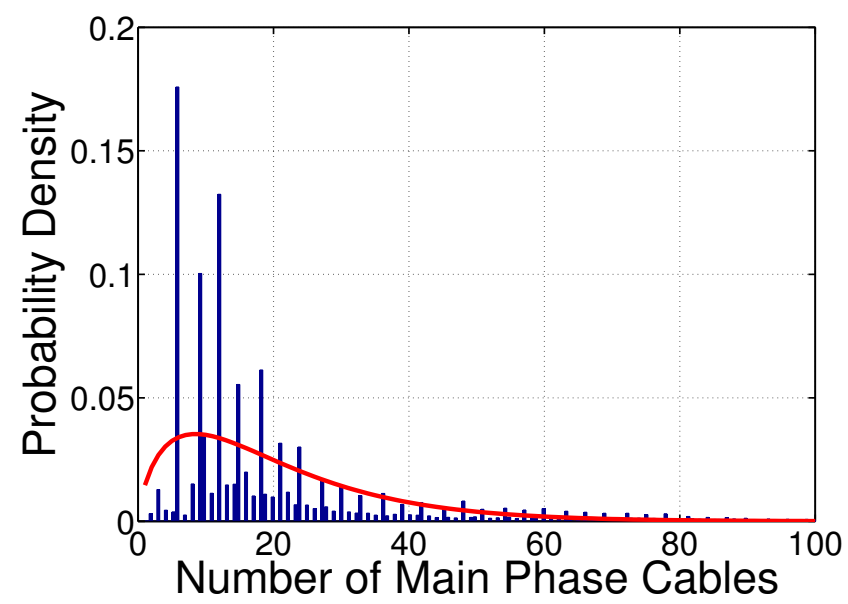

FIG S9. Histogram of number of main phase cables in manholes in Manhattan, along with our fit to a gamma distribution. 
the covariate value was drawn uniformly at random from the range $[0,100]$. Both covariates were normalized afterwards to be between -0.5 and 0.5 .

- Inspection Policy: The schedule of the inspections follows the "bright line" inspection policy over a 5 year period, meaning that each manhole is inspected once every 5 years. This corresponds to $53525 /(365 \times 5) \approx 30$ manhole inspections each day. We also include an ad hoc inspection policy that visits 3 manholes per day on average. Over the simulated 15 year period, our dataset contains 160,575 regular inspections and 16,425 ad hoc inspections.

- Simulation of Events: The events in this dataset are simulated according to the RPP model with the parameters estimated for the Con Edison dataset as given in Section 4.1 of the main paper. For the $g_{2}$ component, we employed the feature-based $\beta$ strategy, where the rate decay was determined by the simulated values of the number of main phase cables and age of oldest cable set, combined using their respective coefficients $v_{1}=-4.6554$ and $v_{2}=-0.5716$. In the dataset, there are a total of 73,758 events.

\section{References.}

Aït-Sahalia, Y., Cacho-Diaz, J. and Laeven, R. J. (2010). Modeling financial contagion using mutually exciting jump processes Technical Report, National Bureau of Economic Research.

Crane, R. and Sornette, D. (2008). Robust dynamic classes revealed by measuring the response function of a social system. Proceedings of the National Academy of Sciences 105 15649-15653.

Egesdal, M., Fathauer, C., Louie, K., Neuman, J., Mohler, G. and Lewis, E. (2010). Statistical and stochastic modeling of gang rivalries in Los Angeles. SIAM Undergraduate Research Online 3 72-94.

Embrechts, P., Liniger, T. and Lin, L. (2011). Multivariate Hawkes processes: an application to financial data. Journal of Applied Probability 48A 367-378.

Engle, R. F. and Russell, J. R. (1998). Autoregressive conditional duration: A new model for irregularly spaced transaction data. Econometrica 1127-1162.

Hawkes, A. G. (1971a). Spectra of some self-exciting and mutually exciting point processes. Biometrika 58 83-90.

Hawkes, A. G. (1971b). Point Spectra of Some Mutually Exciting Point Processes. Journal of the Royal Statistical Society 33 438-443.

Krumin, M., Reutsky, I. and Shoham, S. (2010). Correlation-based analysis and generation of multiple spike trains using Hawkes models with an exogenous input. Frontiers in computational neuroscience 4.

Lewis, E., Mohler, G., Brantingham, P. J. and Bertozzi, A. (2010). Self-exciting point process models of insurgency in Iraq. UCLA CAM Reports $10 \mathbf{3 8 .}$

Louie, K., Masaki, M. and Allenby, M. (2010). A Point Process Model for Simulating Gang-on-Gang Violence Technical Report, UCLA. 
Masuda, N., Takaguchi, T., Sato, N. and Yano, K. (2012). Self-exciting point process modeling of conversation event sequences. arXiv preprint arXiv:1205.5109.

Mohler, G., Short, M., Brantingham, P., Schoenberg, F. and Tita, G. (2011). Self-exciting point process modeling of crime. Journal of the American Statistical Association 106 100-108.

Musmeci, F. and Vere-Jones, D. (1992). A space-time clustering model for historical earthquakes. Annals of the Institute of Statistical Mathematics 44 1-11.

Ogata, Y. (1988). Statistical Models for Earthquake Occurrences and Residual Analysis for Point Processes. Journal of the American Statistical Association 83 9-27.

OgAtA, Y. (1998). Space-time point-process models for earthquake occurrences. Annals of the Institute of Statistical Mathematics 50 379-402.

Reynaud-Bouret, P. and Schbath, S. (2010). Adaptive estimation for Hawkes processes; application to genome analysis. The Annals of Statistics 38 2781-2822.

Rudin, C., Passonneau, R., Radeva, A., Dutta, H., Ierome, S. and Isaac, D. (2010). A Process for Predicting Manhole Events In Manhattan. Machine Learning 80 1-31.

Yan, J., Wang, Y., Zhou, K., Huang, J., Tian, C. H., Zha, H. and Dong, W. (2013). Towards Effective Prioritizing Water Pipe Replacement and Rehabilitation. In Proceedings of the $23^{\text {rd }}$ International Joint Conference on Artificial Intelligence (IJCAI) 2931-2937. IJCAI/AAAI.

S. ERTEKin AND C. RUdin

mit Computer Science and Artificial Intelligence Laboratory

AND

Mit Sloan School of Management

Massachusetts Institute of Technology

Cambridge, MA 02139

E-MAIL: seyda@mit.edu rudin@mit.edu
T. H. MCCORMICK

Department of Statistics

UNIVERSITY OF WASHINGTON

SeAttle, WA 98195

E-MAIL: tylermc@u.washington.edu 